\title{
ATIVIDADE FÁSICA DO SONO REM EM RECÉM-NASCIDOS E LACTENTES E SUA RELAÇÃO COM EPISÓDIOS DE APNÉIA
}

\author{
MAGDA LAHORGUE NUNES*; JADERSON COSTA DA COSTA**; LETICIA TAUFER***
}

\begin{abstract}
RESUMO - A hipótese de que a apnéia pudesse ser o evento final da sindrome da morte súbita da infância (SMSI) deu origem a vários estudos avaliando as características deste evento do ponto de vista polissonográfico. Foi sugerido que a atividade fásica do sono REM estaria associada a ativação dos neurônios respiratórios prevenindo a ocorrência de apnéias prolongadas e protegendo os lactentes de evoluírem para SMSI. Em estudo prévio retrospectivo com recém-nascidos não confirmamos estes achados mas muitos destcs bebês apresentavam comprometimento neurológico severo. O objetivo do presente estudo é verificar a relação existente entre períodos de atividade fásica do sono REM (movimentos oculares rápidos e movimentos de sucção) c apnéia. Estudamos do ponto de vista clínico c polissonográfico 86 reć́m-nascidos c lactentes encaminhados para avaliaçăo por terem apresentado apnéia ou episódios com aparente risco de vida durante o sono. Dividimos os pacientes en 3 grupos: recém-nascidos (idade concepcional inferior a 44 semanas, $n=31$ ), lactentcs com I-6 meses $(n=48$ ) e lactentes com $>6 m<12$ meses $(n=7)$. Todos foram submetidos a avaliação clínica e neurológica para detectar possíveis fatores etiológicos das apnéias. As polissonografias constavam de EEG, eletromiograma submentoniano, eletro-oculograma e monitorização cardiorrespiratória, todas com duração de 2 a 3 horas. Durantc 5 minutos contínuos de sono REM verificamos a incidência de atividade fásica relacionada a períodos em apnéia e comparamos sua incidêncin em pcríodos semelhantes sem apnéia. Nossos resultados mostraram que a atividade fásica do sono REM ocome com maior frequência em períodos sem apnéia. No período neonatal os movimentos de sucção $(p<0.05)$ e nos lactentes com 1-6 meses os movimentos oculares rápidos $(\mathrm{p}=0,001)$. Não houve diferença estatisticamente significativa uros lactentes $>6<12$ meses. Nossos resultados mostram que a atividade fásici do sono REM ć mais frequente cm períodos sem apnéia durante os 6 primeiros meses de vida, período que coincide com o maior risco de SMSI. Sugerimos que o "driving" excitatório que determina o aparecimento da atividade fásica possa ter efeito protetor contra apnéias através do blogueio ou atenuação da inibição tônica do centro respiratório çue determinaria a apnéia. Estudos futuros são necessários para avaliar a relação entre perda desse "mecanismo de proteção"e SMSI.
\end{abstract}

PALAVRAS-CHAVE: apnéia, polissonografia, sîndrome da morte súbita da infância.

The relationship of apnea and phasic activity of REM sleep in newborns and infants that presented an apparent life-threatening event

ABSTRACT - When the apnea hypothesis tumed to be an explanation for sudden infant deall syndrome (SIDS) many authors trierl to find if near-miss or apparent life-threatening events (ALTE) could be markers of SIDS. It was suggested that phasic REM activity is associated with increased respiratory neuron activity and prevents prolonged apnea in near-miss SIDS. In a previous rettospective study with newborns we did not confirm these results, nevertheless we had sevemal babies with serious neurological disturbances. The aim of this study was to verify the relationship of phasic activity (rapid eyes movement and sucking bursts) with both apneic and non-apncic cpochs during REM slecp. We studied 86 childien refered for evaluation because they presented an ALTE during sleep or apnens. The subjects were divided in three groups by age (newborns, $n=31$; $1-6$ months, $n=48$; and $>6<12$ months, $n=7$ ). They were all submitted to a clinical and neurological evaluation in order to detect possible etiological factors to the

Laboratório de Neurofisiologia Clínica do Hospital São Lucas e Faculdade de Medicina da Pontifícia Universidade Católica do Rio Grande do Sul (FM/PUC-RS) Potto Alegre - Brasil. *Prolesssora Adjunta de Neurologia da FM/PUC-RS; ***Professor Titular de Neurologia da FM/PUC-RS; ****Acadêmica da FM/PUC-RS, Bolsista de Iniciaçīo Científica da Fundação de Amapro à Pesquisa do Rio Grande do Sul (FAPERGS). Estudo realizado com apoio da FAPERGS. Aceite: 20-dezcmbro-1996. 
apneic episode. The polysomnograms (PS) consisted of EEG, submental EMG. electro-oculogram and cardiorespiratory monitoring. The records wese made between 11 an and $15 \mathrm{pm}$ for 2 or 3 hours. The electrodes were disposed in the 10-20 system. During one five-minutes epoch of REM sleep we verified the incidence of phasic activity (rapid eyes movement or sucking bursts) time locked to apneic episodes and compared with a similar duration non-apneic epoch. To evaluate the relationship of phasic activity with apneic and non-apneic epochs we compared the incidence of rapicl eyes movements and sucking bursts in each group. Our results showed that phasic activity of REM sleep (sucking bursts) seems to be more frequent in newboms in non-apneic epochs (p $<0.05$ ). Between $1-6$ montlss the number of rapid eyes movements were more frequent in non-apneic epochs $(p=0.001)$. In conclusion our findings suggest that phasic activity of REM sleep seems to be more evident in nonapneic epochs during the first six months of life. Although central apneas may have their origin in an intrinsic disturb of the respirntory control center they may be influenced by the phasic activity of REM sleep. The rapid eyes movements and sucking bursts may have a protection effect against apneas at least in the first 6 months of life, which is the predominant period for SIDS. We suggest that the excitatory driving related to generation of phasic activity in REM slecp may block or atenuate tonic inhibition of respiratory motoneuron and avoid apnea. It is still open for discussion the possible relation between failure of this protective mechanism and SIDS.

KEY WORDS: apnea, polysomnography, sudden infant death syndrome.

Desde 1972 quando Steinschneider ${ }^{23}$ sugeriu que a apnéia prolongada, um componente fisiológico do sono, seria parte da via fỉnal resultante na morte súbita, muitos estudos têm sido desenvolvidos focalizando este evento. Os bebês que apresentavam episódios de risco de síndrome da morte súbila da infância (SMSI) eram anteriormente denominados de "near miss", um termo até hoje sem tradução. Como este nome deixa implícita uma relação com a SMSI que nem sempre demonstra ser a evolução destas crianças, ele foi substituido por "ALTE - apparcnt life-threatening event" ou eventos que aparentemente trazem risco de vida. Estes eventos podem ser descritos como episódio que aterroriza o observador sendo caracterizado pela combinação de apnéia, alteração da coloração da pele ou mucosas, hipotonia, choque ou engasgo?. A hipótese de episódios de ALTE poderem scr marcadores de alto risco para SMSI explica o grande número de pesquisas que vêm sendo desenvolvidas neste grupo de crianças. A incidência de ALTE na populacāo geral ć aproximadamente 0,5 a $6 \%$. Após ampla investigação é encontrada etiologia específica para estes cventos em mais ou menos $50 \%$ dos $\operatorname{casos}^{2.9}$. Durante o sono REM os motoncurônios do tronco cerebral estão touicamente hiperpolarizados (inibidos) e os músculos por eles inervados tornam-se hipotônicos. Exceto pelos breves episódios fásicos de movimentos oculares rápidos (MOR), abalos muscularcs corporais c atividade respiratória, os restantes motoneurônios estāo inibidos. As ondas ponto-genículo-occipitais (atividade fásica dos MOR) e a atonia postural (atividade tônica) parecem ter origem em grupos celulares distintos da formação reticular dorso-lateral ponto-mesencefálica ${ }^{\mathrm{x} .22}$. Watanabe el al. (1983) sugeriram que a atividade fásica (MOR) do sono REM cstaria associada a aumento da atividade dos neurônios respiratórios e preveniriam a ocorrência de apnéias prolongadas durante o sono, evitando a ocorrência de $\mathrm{SMSI}^{24}$. Em estudo retrospectivo posterior não confirmamos estes resultados, mas nossa casuística era composta somente de recém-nascidos (RN) c alguns apresentavam patologias neurológicas graves ${ }^{4}$.

O objetivo do presente estudo é verificar, cm RN c lactentes que apresentaram apnéias ou ALTE, a relação entre atividade fásica do sono REM (MOR e movimentos de sucção) c períodos de apnéia na tentativa de estabelecer parâmetros polissonográficos que indiquem risco de SMSI.

\section{METODOLOGIA}

Estudo retrospcctivo em que foram revisadas as polissonografias (PS) de 86 crianças encaminhadas para avaliação por relato de apnéia ou por terem apresentado episódio de ALTE. Os pacientes forann divididos cm 3 grupos de acordo com a faixa etária : grupo 1, RN com idade concepcional igual ou inferior a 44 semanas (n=31); grupo 2, lactentes com 1-6 meses ( $n=48$; c grupo 3, lactentes com 9-11 meses $(n=7)$. Além da avaliaçĩo polissonográfica estas crianças foram submetidas a investigação clínica e neurológica no sentido de detectar fatores etiológicos para os referidos episódios. Não foram incluídos no grupo I, RN com diagnóstico de apnéias convulsivass. 


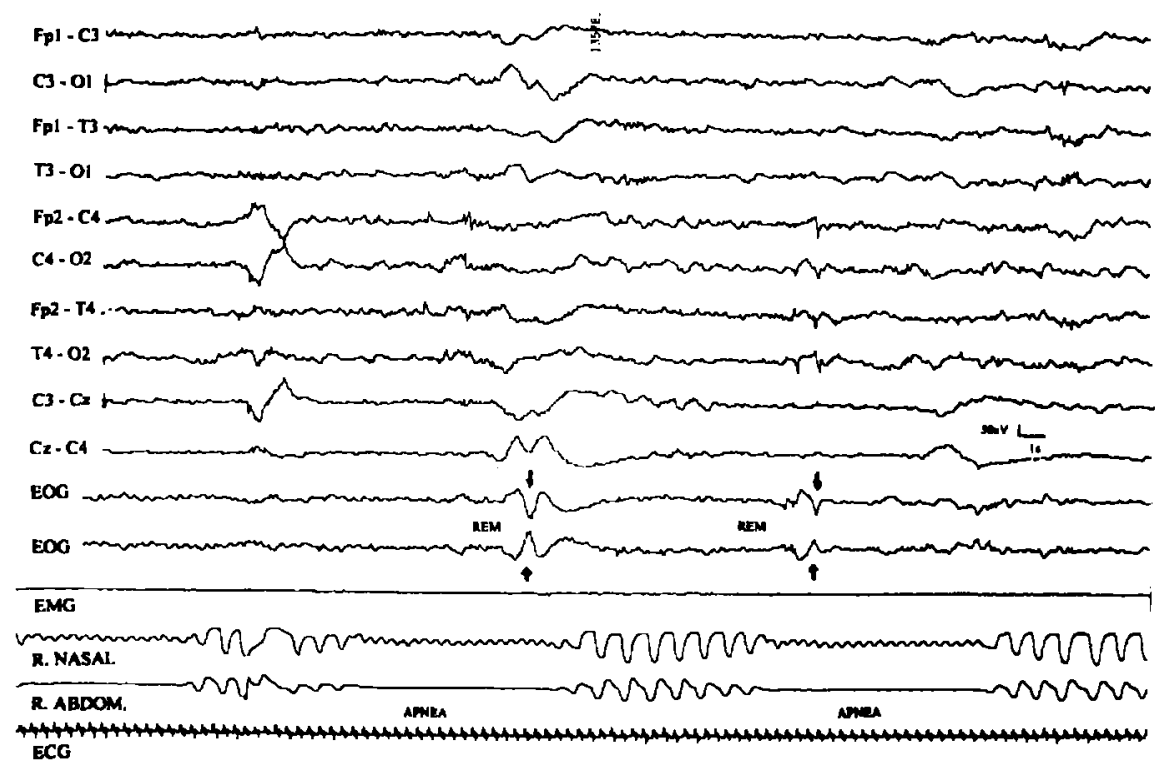

Fig 1. O registro polissonográfico evidencia movimentos oculares rápidos coincidentes aos períodos em apnéia.

As PS foram realizadas em polígrafos de 8, 13 ou 16 canais e consistiam de eletrencefalograma (EEG), eletromiograma submentoniano (EMG), eletro-oculograma (EOG) e monitorização cardiorrespiratória. Os registros foram realizados no horário entre 11:00 e 15:00 $\mathrm{h}^{7}$ por periodos de 2 a 3 horas. A colocaçāo de eletrodos foi realizada de acordo com o sistema 10-20 (nos RN sistema 10-20 modificado para o periodo neonatal) e a resistência foi mantida abaixo de $5 \mathrm{kOhm}{ }^{1.3}$.

O estado comportamental foi classificado em 4 estágios $^{1.7^{2}}$ : acordado (olhos abertos ou chorando, piscamento, movimentos corporais), sono REM (olhos fechados, atividade fásica: MOR identificados no EOG e/ou movimentos de sucção identificados no EMG, movimentos corporais, componente afetivo: choro, sorriso ou careta e respiração irregular), sono NREM (olhos fechados, movimentos oculares lentos, respiraçāo regular) e sono transicional (estado em que os critérios para sono REM e NREM não são totalmente preenchidos).

En cada PS foram identificados: no grupo I periodos de sono REM, NREM e transicional; nos demais grupos períodos de sono REM e NREM ${ }^{1,3,1 \times}$. Durante 5 minutos consecutivos de sono REM a incidência de atividade fásica (MOR e movimentos de sucção) foi verificada concomitantemente aos episódios de apnéia e no primeiro periodo subsequente, com duraçāo semelhante, em que a atividade respiratória estava regular. Consideramos somente episódios de apnéia com duraçāo superior a 3 segundos. Neste estudo foi avaliada a primeira PS de cada paciente. isto é, prévia ao início do tratamento (Figs 1 e 2).

Para avaliar a relação entre atividade fásica e períodos come sem apnéia, comparamos a incidência de MOR e movimentos de sucçāo nestes períodos utilizando teste estatístico paramétrico (teste para diferença de médias de amostras relacionadas) em que obtivemos as distribuições de probabilidade zc e tc. Consideramos as diferenças estatisticamente significativas para o valor de $p<0,05$.

\section{RESULTADOS}

O grupo 1 consistiu de 31 RN com idade gestacional entre $32-40$ semanas, 11 prematuros e $20 \mathrm{RN}$ a termo. A idade concepcional na data da realização da PS variou entre 36 e 44 semanas,sendo 15 masculinos e 16 femininos. Além do episódio de apnéia um RN apresentou crises convulsivas possivelmente associadas a asfixia perinatal moderada e outros dois, mioclônias benignas do sono. Outras etiologias relacionadas ao quadro de apnéia foram diagnosticadas em $3 \mathrm{RN}$ : síndrome de Beckwith-Wiedemann, cardiopatia congênita e doença da membrana hialina. A avaliação clínica e 


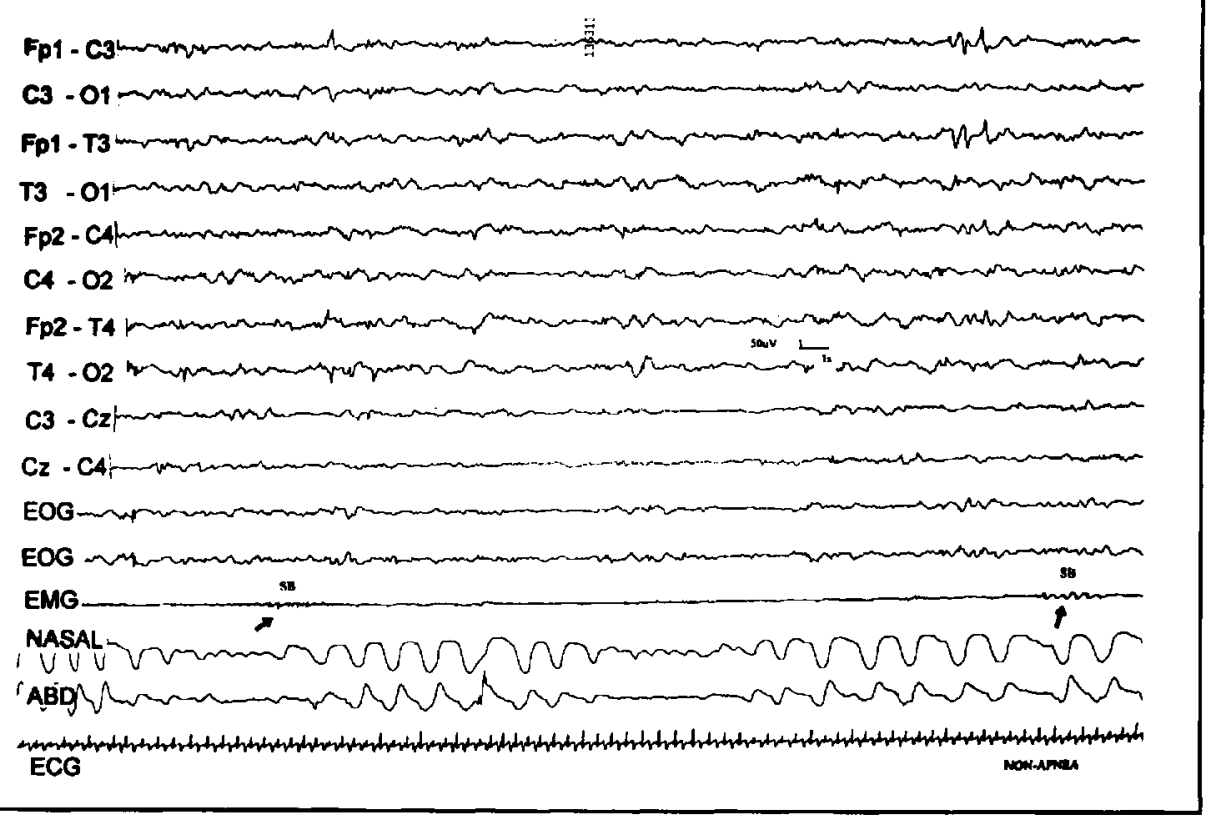

Fig 2. O registro polissonográfico apresenta movimentos de sucção em períodos sem apnéia.

neurológica foi normal nos outros RN. Um RN tinha irmão com diagnóstico de SMSI. Os EEG foram normais em $25 \mathrm{RN}$. Os achados anormais nos exames restantes foram: em cinco casos padrão de "dismaturidade" e em um caso padrão multifocal". O tempo total em apnéia durante os cinco minutos consecutivos de sono REM variou entre 0 e 209 segundos (média $=57,19$ e desvio padrão= 48,51). O número de MOR em períodos com apnéia variou de 0 a 14 (média $=1,81$ e desvio padrão= 3,02 ) e de movimentos de sucção variou entre 0 e 5 (média $=1,22$ e desvio padrão=1,65). No período sem apnéia o número de MOR variou de 0 al 8 (média=3,39 e desvio padrão=4,90), 0 número de movimentos de sucção variou de 0 a 27 (médja=2,36 e desvio padrão=5,47). Comparando os movimentos de sucção nos períodos com duração semelhante com e sem apnéias obtivemos diferença estatisticamente significativa $(t c=2,10, p<0,05)$, houve maior número de movimentos de sucção nos períodos sem apnéia.

O grupo 2 consistiu de 48 RN com idade entre 1 mês e 6 dias e 6 meses, 9 foram prematuros, 23 eram do sexo masculino e 25 feminino. A investigação clínica neste grupo mostrou 10 lactentes com refluxo gastroesofágico, um com tetralogia de Fallot, um com microcefalia e um caso de síndrome de Dandy-Walker. Um lactente tinha irmão com diagnóstico de SMSI. Os EEG foram normais em 42 pacientes, focal $\mathrm{em}$ cinco e multifocal em um caso. Durante cinco minutos consecutivos de sono REM o tempo total em apnéia variou de 0 a 177 segundos (média= 31,01 e desvio padrão $=30,77$ ), o número de MOR variou de 0 a 19 (média $=1,4$ e desvio padrão=3,15) e o número de movimentos de sucção variou de 0 a 17 (média $=0,98$ e desvio padrão $=1,62$ ). No período sem apnéia o número de MOR variou de 0 a 18 (média=2,71 e desvio padrão=4,09) o número de movimentos de suç̧ão variou de 0 a $16($ média $=1$ e desvio padrão=2,72). O número de MOR foi significativamente superior nos períodos sem apnéia.

O grupo 3 consistiu de 7 lactentes com idade entre 9 e 11 meses, 4 do sexo masculino e 3 do feminino. A investigação etiológica mostrou refluxo gastroesofágico em dois casos, crise convulsiva associada a policitemia sintomática em um caso e epilepsia do lobo temporal (diagnosticada 
Tabela 1. Atividade fásica do sono REM em períodos com e sem apnéia.

\begin{tabular}{lcc}
\hline Atividade fásica & Apnéiil & Sem apnéia \\
\hline Grupo I & & \\
MOR & $1,8 \pm 3,02$ & $3,3 \pm 4,90$ \\
MS & $1,2 \pm 1,65$ & $2,3 \pm 5,47^{*}$ \\
TTA & $57,1 \pm 48,51$ & - \\
Grupo II & & $2,7 \pm 4,09^{* *}$ \\
MOR & $1,4 \pm 3,15$ & $1,0 \pm 2,72$ \\
MS & $0,9 \pm 1,62$ & - \\
TTA & $31,01 \pm 30,77$ & $0,8 \pm 1,07$ \\
Grupo III & & 0 \\
MOR & $1,1 \pm 1,07$ & - \\
MS & $0,5 \pm 1,00$ & \\
TTA & $15,9 \pm 16,78$ & \\
\hline
\end{tabular}

MOR, movimento ocular rápida; MS, movimento de sucção; TTA, tempo total em apnéia; *tc=2,10 e p<0,05; ** zc=-3,1 e p=0,001.

posteriormente) em um caso. Os EEG foram normais em todos os pacientes. Durante cinco minutos consecutivos de sono REM o tempo total em apnéia variou de 0 a 50 segundos (média=15,9 e desvio padrāo 16,78), o número de MOR variou de 0 a 3 (média=1,14 e desvio padrão=1,07) e o número de movimentos de sucçāo variou de 0 a 2 (média $=0,5$ e desvio padrão=1). No período sem apnéia o número de MOR variou de 0 a 3 (média= 0,86 e desvio padrão=1,07) e não foram registrados movimentos de suç̧ão. Não houve diferença estatisticamente significativa na incidência de atividade fásica em relação aos períodos com e sem apnéia.

O tempo total em apnéia diminuiu com o aumento da idade dos pacientes assim como o registro de movimentos de sucção que é típico do periodo neonatal. Os resultados obtidos nos três grupos estudados estão descritos na Tabela 1. No seguimento nenhuma destas crianças evoluiu para SMSI.

\section{DISCUSSÃO}

Por definição caracteriza-se a SMSI como a morte súbita e inexplicada, mesmo após extensa investigação, de lactente com idade inferior a um ano. Estudos epidemiológicos, no entanto, demonstram entre outras características, que na maioria dos casos a morte ocorre entre 1 e 5 meses, consideramos então este período o de maior risco". Já os episódios de ALTE ocorrem em população mais heterogênea sendo possível em até $50 \%$ dos casos chegarmos a um diagnóstico específico. Os estudos em geral dividem os pacientes em dois grupos para fins de seguimento: ALTE sintomático (em que diagnóstico específico foi realizado) e ALTE idiopático (ou apnéia do sono da infância). A mortalidade dos episódios de ALTE sintomático é de aproximadamente $1 \%$ aumentando nos caso de ALTE idiopático para 3\%. A causa prcdominante de morte nas crianças com ALTE é a SMSI, entretanto a maioria dos lactentes com SMSI nunca havia apresentado episódio anterior de ALTE. Nenhum dos nossos casos de ALTE apresentou SMSI no seguimento.

A perspectiva de detectar fatores relacionados ou preditivos de risco da SMSI tem impulsionado a realização de diversos estudos ${ }^{2,4,7,9,23,24}$. A tentativa de estabelecer parâmetros de normalidade para as apnéias e respiração periódica, eventos frequentes nos $\mathrm{RN}$, através de fórmulas diversas (como indices e densidade entre outros), tem também motivado o desenvolvimento de muitos estudos ${ }^{\text {h.1111.17.21. }}$ 
Observamos diminuição do tempo total em apnéia relacionado com o aumento da idade, isto é, do grupo 1 para o grupo 3 houve redução de $73 \%$. Este é um achado fisiológico já observado $\mathrm{cm}$ estudos anteriores ${ }^{6,17}$ em que a ceasuística era formada por RN. Nosso estudo amplia csta observação até o final do primciro ano de vida.

A densidade de MOR ê indice rotineiramente utilizado nas polissonografias para avaliação da atividade fásica durante o sono REM, sendo que esta densidade se mantem constante mesmo com a redução fisiológica do sono REM que ocorre em adultos ${ }^{20}$. $\mathrm{O}$ cálculo de densidade de MOR não foi utilizado em nosso estudo, mas o número absoluto de MOR se manteve constante entre os grupos.

Para gerar atividade muscular fásica (movimentos horizontais dos olhos e movimentos de sucção) acredita-se que seja necessária forte excitação motora que se sobreponha à inibição do motoneurônio. Excitação $\mathrm{c}$ inibição do motoneurônio são originadas no tronco cerebral assim como a geração da atividade fásica do sono $\mathrm{REM}^{\text {.15. }}$. Disfunção da inibição tônica ocorre cm patologias do tronco e ponte $\mathrm{s}^{\mathrm{s} 15}$. Kohyama et al. publicaram diversos estudos ${ }^{11-16}$ demonstrando a possibilidade de avaliar, atravćs da polissonografia, o amadurecimento do tronco cerebral calculando-se índices de atividade muscular fásica. Os autores propōem dois índices: 1. índice de dissociação (movimentos de suç̧ão com duração interior a 5 segundos/ $m s<5 s+m s>5 s$ ) e 2 . percentual de movimentos breves (\% de $\mathrm{ms}<5 \mathrm{~s}$ que ocorrem simultaneamente aos surtos de movimentos oculares rápidos). $\mathrm{O}$ índice de dissociação aumenta com a idade concepcional $\mathbf{c}$ avalia a maturidade do sistema tônico inibitório. O percentual de movimentos breves diminui com a idade concepcional e reflete a maturidade da atividade fásica. Watanabe et al. avaliaram através de polissonografia RN com cpisódios frequentes de apnéia, observaram que estes episódios geralmente não ocorriam associados a MOR. Sugerem que o sono REM é um estágio vulnerávcl ao acontecimento de SMSI c que os surtos de MOR observados teriam ação facilitadora sobre os neurônios respiratórios, protejendo os RN da SMSI ${ }^{24}$. Este estudo apresenta erro metodológico, pois os autores considcraram a incidência de MOR em períodos com e sem apnéia de duração diversa. Como a duraçāo dos períodos sem apnćia ć superior à dos períodos em apnéia, existia maior probabilidade da ocorrência de MOR nos períodos sem apnéia.

Em nosso cstudo avaliamos os dois tipos de atividade fásica registrados em sono REM comparando períodos em apnéia e sem apnéia com duração semelhante. Nos grupos 1 e 2, cujos pacientes se cncontram na faix a etária de maior risco para SMSI, observamos aumento da incidência de atividade fásica nos periodos scm apnéia comparativamente a períodos semelhantes em apnéia, com diferença cstatisticamente significativa.

Estes achados sugerem que a atividade fásica do sono REM, caracterizada por movimentos de sucção e MOR, parece ter um efeito protetor contra as apnéias. Sugerimos que o "driving" excitatório que determina o aparecimento dos movimentos fásicos, mediados pelo tronco cerebral, possa bloquear ou atenuar a inibição tônica do centro respiratório que determinaria a apnéia. São necessários estudos futuros para avaliar a possível relação entre a perda desse mecanismo aparentemente protetor c SMSI.

\section{REFERÊNCIAS}

1. Anders T. Emde R, Parmelee A. A minual of standardjzed terminology, techniques and criteriat for scoring states of sleep and wakctulness in newborn infants. UCLA Brain Service, Los Angeles: BRI Publications Office, 1971.

2. Brooks JG. Apparent life-threatening events and apnea of infancy. In Hunt CE (ed). Apnea and SIDS. Philadelphial: WB Samuders, 1992:809-838.

3. Da Costa JC. O sono cın recém-nascidos: aspectos polissonogrífícos. In Reimăo R (ed). Sono aspectos attuais. São Paulo: Atheneu, 1990:133-16I.

4. Da Costa JC. Nunes ML, Wagner EM. A prospective study of the apneas and its relationship with the plassic activity of REM sleep in Newborns: clinical and polygraphic aspects. Electroenceph Clin Neurophysiol 1980;75:531.

5. Da Costa IC, Nunes ML, Nori DB, Lopes DK, Perondi G. Fugundes SC. Apneic seizures in newbons. Pediatr Neurol 1992:8:382.

6. Flores-Guevata R. Plouin P, Curzi-Discalova L, Radvanyi MF, Guidasci S, Pajot N, Monod N. Sleep apmeas in normal neonates and infants during the first 3 months of life. Neuropediatrics 1982;13(Suppl):21-28. 
7. Guilleminaut C. Atiago R, Korobkin R, Coons S, Owen-Boediker M, Baldwin R. Sleep paraneters and respilatory vilriables in near miss sudden infant deith syndrome infants. Pediatrics $1981 ; 68: 354-360$.

8. Guilleminaut C. Baker TL. Sleep and its disorders. In Asbury K, Mc Khann GM, McDonald WI (eds). Disorders of the nervous system clinical neurobiology. Philadelphia: WB Silunders 1986:867-879.

9. Hoffman H.I. Hillman LS. Epideniology of the sudden infant death syndrone: maternal, nconattal and postheonatal risk liators. In Hunt CE (cel). Apnea and SIDS. Philadelphia: WB Siunders, 1992:717-737.

10. Kelly IH. Shannon DC. Periodic breathing in infants with near-miss sudden intant deatl synclrome. Pediatrics $1979 ; 63: 355-360$

11. Kohyama J. Iwakitwil Y. Polygraphic features of a victim of sudden intant death syndrome and of infants with alppillent lifethreatening event. Brain Dev 1989;11:186-90.

12. Kohyania J, Iwakawa Y. Developmental changes in phasic sleep parameters as reflections of the blain-stem maturition: polysomnogritphical examinations of infants, including premature neonates. Electroenceph Clin Neuropluysiol 1990;76:325-330.

13. Kohya!ma J, I wikawa Y. Intenelationships between rupid eyc and body movements during sleep: polysomnographic examinations of intants including prensature neonates. Electroenceph Clin Neurophysiol 1991;79:277-280.

14. Kohyama J. Masayuki S, Itoh M, Fukumizu M, Iwakawa Y. Phasic muscle activity during REM sleep in infancy: nornal mutturation and contrastive abnomality in SIDS /ALTE and West syndrome. J Sleep Res 1993:2:241 -249.

15. Kohyima J. Masilyuki S, Yoshibide 1 . Brainstem control of phasic muscle activity during REM slecp: a revicw and hypotlesis. Britin Dev 1994: [6:81-9!.

16. Kohyama J, Shimohiriı M, Hasegawa T, Kouji T. Iwakawa Y. Phasic motor activity reduction occurring with horizontal rapid eye movements during active sleep in human. Exp Bruin Res 1995;107:137-144.

17. Lee D. Caces R. Kwiatkowski K, Cites D. Rigatto H. A developmental study on types and frequency distribution of shout apueas in term and preterm infants. Pediatt Res 1987;22:344-349.

18. Niedermeyer E. Maturation of the EEG: development of waking and sleep patterns. In Niedermeyer L. Lopes cla Silva F (eds). Electroencephalography. Baltinore-Munich: Urban \& Schwarzenberg. 1987:133-157.

19. Nunes ML, Da Costa JC, Roitman I, Fernandes RMF. Guia técnico para execução de registro poligräfico e eletrencefialoğranıa no periodo neonatal. BJECN 1996:2:27-42.

20. Reimāo R, Lemmi H, Hamlet III J. Sander C, Zwáag RV. Densidade dos movimentos oculares rápidos durante o sono alivo em recén-nascidos pré-termo. Arq Neuropsiquiatr 1986;44:219-233.

21. Rigutto H. Brady J. Periodic breathing and apnea in preterm infants: evidence for hypoventilation possibly due to central respiratory depression. Pediatrics 1972:50:202-218.

22. Sakail K. Some anatomical ind physiological properties of ponto-mescncephalic tegmental neurons witl special reference to tlu: PGO waves and postural atonial daning paradoxal sleep in call. In Hobson JA, Brazier MAB (eds). The reticular formation revisited. New York: Raven Press, 1980:427-447.

23. Steimschmeider $\Lambda$. Prolonged apnea and the sudden infant death syndrome: clinical and laboratory nbservations. Pediatriis 1972;50:646.

24. Watambe K, Inokuma K. Negoro T. Rem sleep prevents sudden intant death syndrome. Eur I Pediatr 1983; 140:289-292. 